\title{
BUNCH LENGTH MEASUREMENTS USING A MARTIN PUPLETT INTERFEROMETER AT THE TESLA TEST FACILITY LINAC
}

\author{
B. Leissner, Ch. Berger, R. Siedling, M. Tonutti, RWTH Aachen, D-52056 Aachen \\ M. Geitz, G. Schmidt, P. Schmüser*, DESY, D-22603 Hamburg
}

\section{Abstract}

The longitudinal charge distribution of the electron bunches in the TESLA Test Facility linac has been determined using coherent transition radiation produced at a thin metal foil. The autocorrelation of the radiation pulse is measured with a Martin Puplett interferometer and then Fourier transformed to yield the coherent radiation spectrum and the bunch form factor. Several techniques, including a Kramers-Kronig analysis, have been applied to determine the longitudinal bunch charge distribution. Measurements with a thermionic electron gun and a radio-frequency photo injector are presented. The influence of frequencydependent acceptance corrections is discussed.

\section{INTRODUCTION}

Future electron drive linacs for Free Electron Lasers in the VUV and X ray regime require the production and acceleration of bunches whose length is in the sub-picosecond regime [1]. Coherent Transition Radiation (CTR) emitted by the electron bunches upon crossing a thin aluminum foil can be used to determine the longitudinal charge distribution. The radiator is oriented at an angle of $45^{\circ}$ with respect to the beam direction so that the radiation can be extracted at $90^{\circ}$ through a quartz window. The spectral intensity emitted by a bunch of $N$ particles consists of an incoherent and a coherent term

$$
I_{t o t}(\omega)=N I_{1}(\omega)+N(N-1) I_{1}(\omega)|f(\omega)|^{2} .
$$

Here $I_{1}(\omega)$ is the intensity radiated by a single electron at the frequency $\omega$ and $f(\omega)$ is the bunch form factor [2, 3, 4], defined as the Fourier transform of the normalized charge distribution $\rho$. For a relativistic bunch whose transverse dimensions are small compared to the length the form factor becomes

$$
f(\omega)=\int \rho(z) \exp (i \omega z / c) d z=\int c \rho(c t) \exp (i \omega t) d t .
$$

\section{THE MARTIN PUPLETT INTERFEROMETER}

The Martin Puplett interferometer used to measure the autocorrelation function of the radiation pulse is shown schematically in Figure 1. The interferometer has been fabricated at the physics institute of the RWTH Aachen according to optical standards and is rigidly mounted on a

\footnotetext{
* permanent adress: University of Hamburg, D-20146 Hamburg
}

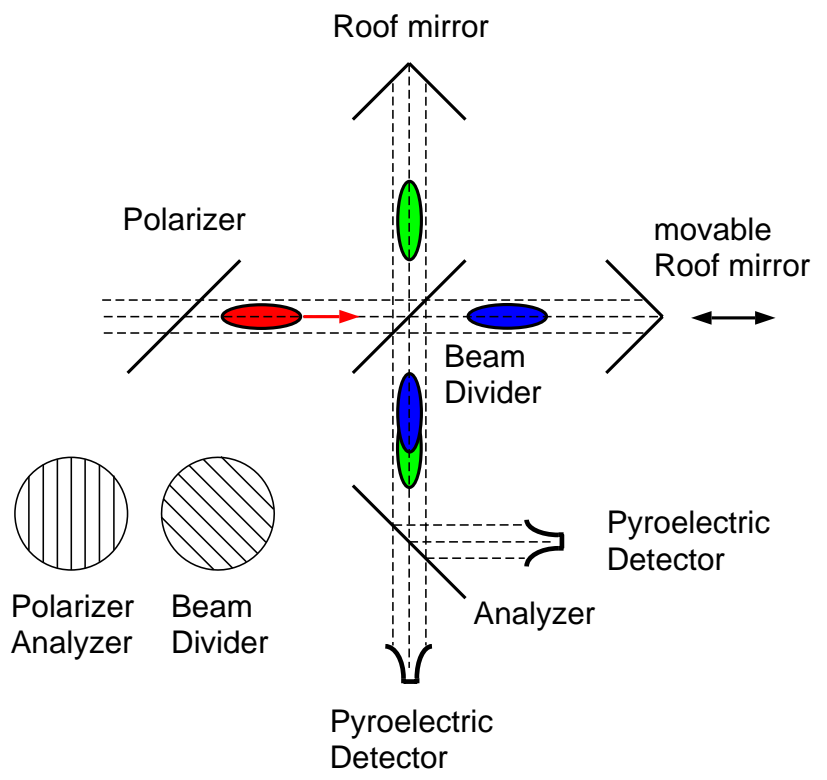

Figure 1: The Martin Puplett interferometer.

$40 \mathrm{~mm}$ thick aluminum plate. Wire grids wound from 20 $\mu \mathrm{m}$ diameter thick gold plated tungsten wire with a spacing of $100 \mu \mathrm{m}$ act as polarizer, beam splitter and analyzer. The incident radiation pulse is polarized horizontally by the first grid and then splitted by the beam divider into components of different polarization entering the two spectrometer arms. The polarization is flipped by the roof mirrors, hence the component first transmitted at the beam splitter is now reflected and vice versa. The recombined radiation is in general elliptically polarized, depending on the path difference in the two arms. The analyzing grid transmits one polarization component into detector 1 and reflects the orthogonal component into detector 2. Two pyroelectric detectors equipped with horn antennas are used as detection devices for the sub-millimeter wavelength radiation. Not shown in the schematic diagram are two parabolic mirrors of optical quality which transform the diverging beam leaving the CTR radiator into a parallel beam and focus the recombined beam onto the detectors.

The result of a scan is shown in Figure 2. Plotted is the response of the two detectors as a function of the time delay between the two spectrometer arms. Both detectors register an autocorrelation signal but with opposite sign. The sum of the two detector signals is a measure of the total radiation power entering the interferometer. The raw data show some fluctuations which occur simultaneously 


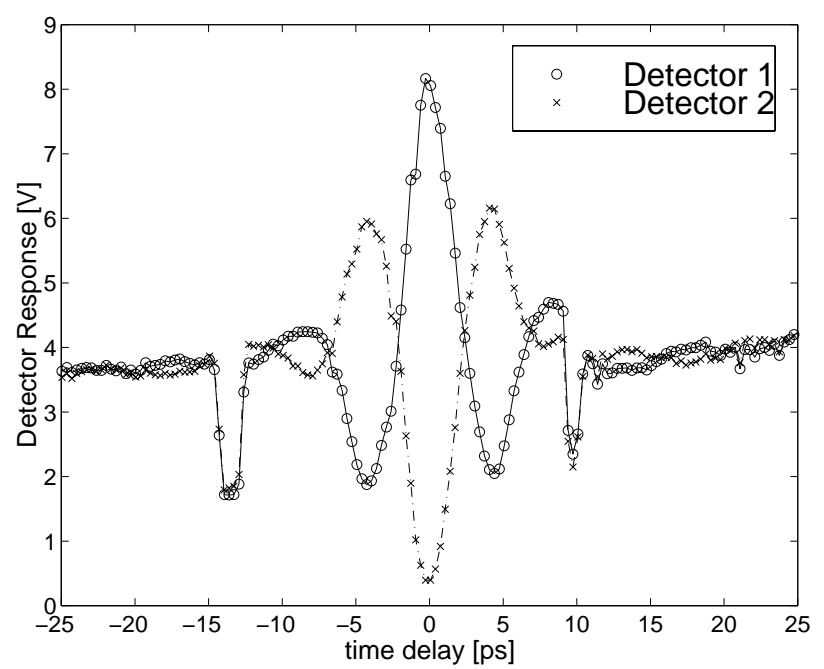

Figure 2: The signals of the two pyroelectric detectors (circles and crosses) as a function of the path difference in the two spectrometer arms. Note the anticorrelation in the interference signals. The correlated fluctuations at $-13 \mathrm{ps}$ and +9 ps are due to intensity fluctuations of the electron beam.

in both detectors and can be attributed to instabilities in the electron beam. Such correlated fluctuations can be eliminated by taking the difference of the two detector signals and normalizing it to the sum. The normalized difference interferogram, presented in Figure 3, shows in fact a very smooth behaviour. Remaining statistical fluctuations due to amplifier noise etc are within the size of the data points.

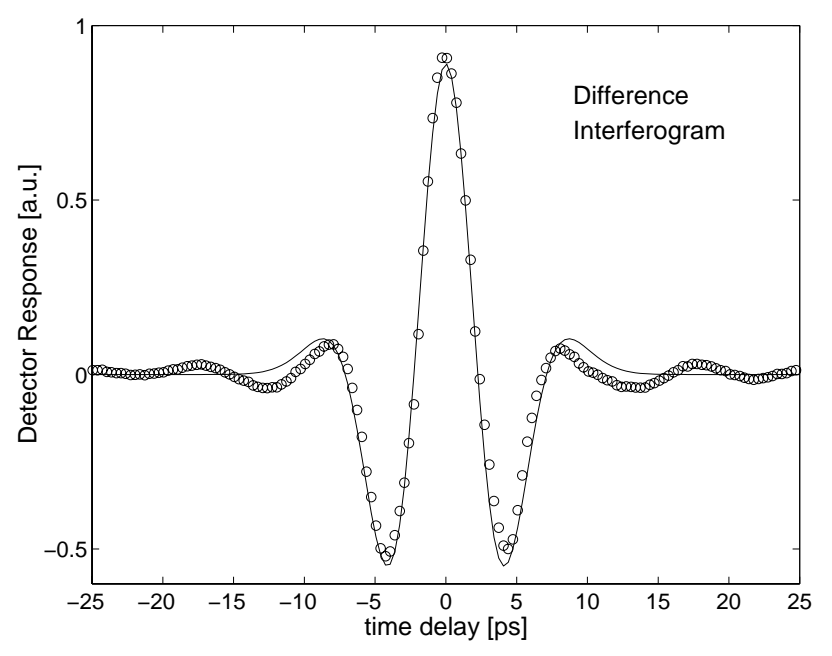

Figure 3: The difference of the signals of the pyroelectric detectors normalized to the sum (open circles). The solid curve is the result of a time domain fit explained in section 3.1. The data were taken with a thermionic cathode.

\section{DATA ANALYSIS}

The power spectrum of the coherent transition radiation is obtained by Fourier transformation of the autocorrelation

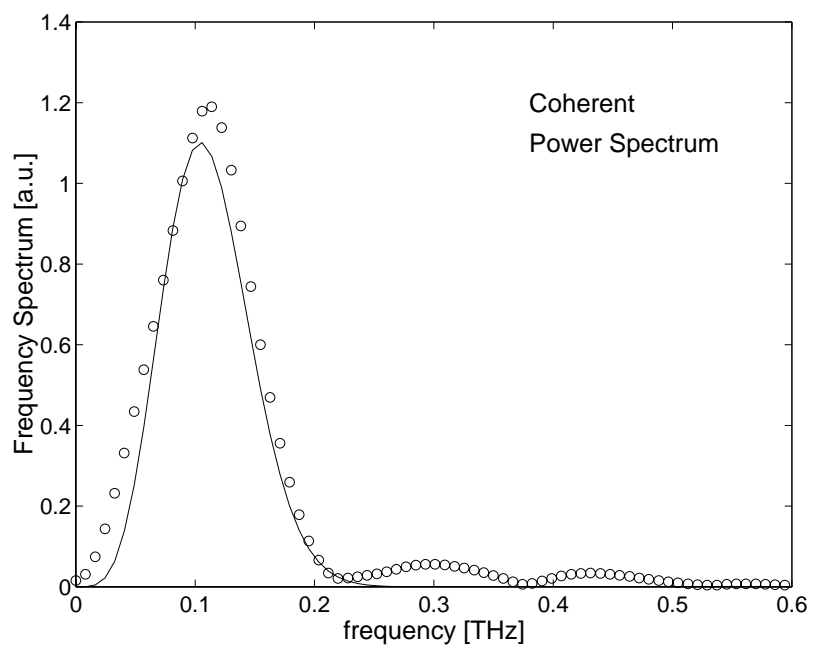

Figure 4: The power spectrum corresponding to the interferogram of Figure 3 (open circles). The suppression at low frequencies is due to the high-pass characteristics of the experimental setup. The solid curve is the frequency domain equivalent of the time domain fit explained in section 3.1.

function. The resulting power spectrum is shown in Figure 4. The power spectrum reveals one of the main limitations in the application of frequency-domain techniques for determining the bunch length: the low frequency part of the spectrum is strongly suppressed. Several effects contribute: diffraction losses due to the limited apertures in the interferometer, the finite size of the radiator foil which leads to a widening of the angular distribution of transition radiation at low frequency, and also the reduced acceptance and sensitivity of the detectors at long wavelengths. These systematic effects are still under study. In the following analysis they will be treated in an empirical way only, and the resulting bunch lengths have to be taken with some care.

\subsection{Time Domain Fitting}

The low frequency part of the power spectrum is suppressed by cutoff effects. Following Murokh et al. [5] we describe this in terms of a filter function

$$
g(\omega)=1-e^{-\xi^{2} \omega^{2}}
$$

where $\xi$ is an adjustable parameter. Assuming a Gaussian longitudinal charge distribution of variance $\sigma$, the measured power spectrum is expected to be

$$
\tilde{S}(\omega)=e^{-\sigma^{2} \omega^{2}}\left(1-e^{-\xi^{2} \omega^{2}}\right)^{2}
$$

Transforming to the time domain we obtain the expected autocorrelation curve

$$
S(t)=\left(e^{-\frac{t^{2}}{4 \sigma^{2}}}-\frac{2 \sigma \cdot e^{-\frac{t^{2}}{4\left(\sigma^{2}+\xi^{2}\right)}}}{\sqrt{\sigma^{2}+\xi^{2}}}+\frac{\sigma \cdot e^{-\frac{t^{2}}{4\left(\sigma^{2}+2 \xi^{2}\right)}}}{\sqrt{\sigma^{2}+2 \xi^{2}}}\right)
$$


The result of the fit of Equation (5) to the interferogram is presented as the continuous curve in Figure 3. The obtained $\mathrm{rms}$ bunch length is $\sigma=2 \mathrm{ps}$. The error of the analysis is dominated by the systematic low-frequency effects and is estimated to be in the order of $20 \%-30 \%$.

\subsection{Kramers Kronig Analysis}

A Fourier transformation of the autocorrelation function yields only the absolute magnitude $|f(\omega)|$ of the form factor. The Kramers-Kronig dispersion relation approach can be used to compute the phase. The so-called minimal phase $\psi$ is given by [6]

$$
\psi(\omega)=\frac{-2 \omega}{\pi} \int_{0}^{\infty} \frac{\ln [|f(u)| /|f(\omega)|]}{u^{2}-\omega^{2}} d u
$$

The inverse Fourier transformation yields then the desired longitudinal charge distribution

$$
\rho(z)=\int_{0}^{\infty}|f(\omega)| \cos (\psi(\omega)+\omega z / c) d \omega
$$

The Kramers Kronig technique will be applied to the interferogram in Figure 5. The low frequency attenuation of the frequency spectrum has been corrected for by smoothly extrapolating the form factor with a fourth order polynomial. The reconstructed bunch form, depicted in Figure 6 , is trapezoidal with a FWHM of about 4 ps. It should be noted, however, that the result of the reconstruction depends strongly on the exact parametrization and the starting point and slope of the extrapolation. It is possible to obtain differences of up to $1 \mathrm{ps}$ in FWHM and variations of the shape by varying the initial conditions and the parametrization of the extrapolation.

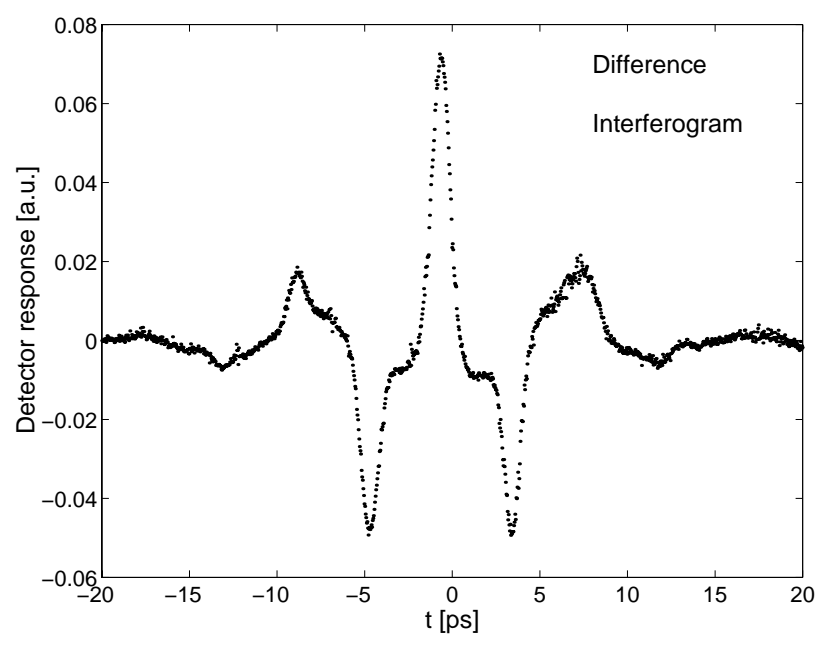

Figure 5: Normalized difference interferogram measured at the TTF linac equipped with an rf photo-cathode and a bunch compressor.

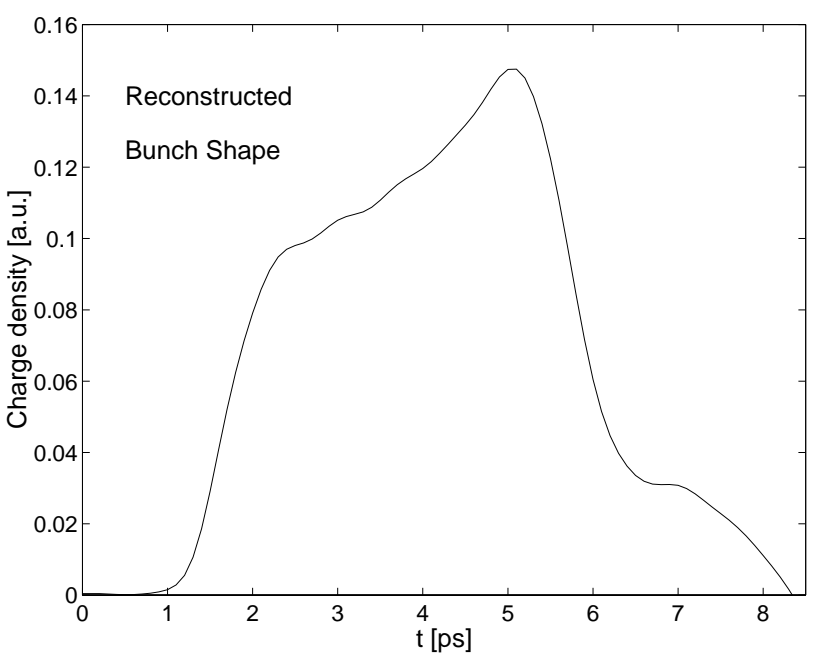

Figure 6: Longitudinal bunch profile as reconstructed by the Kramers Kronig technique.

\section{CONCLUSION}

A high precision Martin Puplett interferometer has become a standard device for bunch length measurements at the TESLA Test Facility linac. One useful side effect is that the signal amplitude of the pyroelectric detectors increases strongly with decreasing bunch length and can therefore be used as a fast monitor for optimizing the bunch compression in the linac. Bunch lengths and bunch shapes have been determined for various machine configurations. The main problem for precise measurements of picosecond bunches is the not-well known low frequency attenuation of the coherent radiation. Detailed investigations are in progress.

\section{REFERENCES}

[1] TESLA - Collaboration, DESY-TESLA 95-01 (1995).

[2] C. J. Hirschmugl et al. , Physical Review A, Vol. 44, No. 2 (1991).

[3] J. S. Nodvick, D. S. Saxon, Physical Review, Vol. 96, No. 1 (1954).

[4] E. B. Blum, U. Happek, A. J. Sievers, Nucl. Instr. Meth. A307 (1991).

[5] A. Murokh, J. B. Rosenzweig, U. Happek, M. Hogan, H. Suk, G. Travish, Phys. Rev. Lett., (1998).

[6] R. Lai, A. J. Sievers, Nucl. Instr. Meth. A 397, (1997), 221 231.

[7] B. Leissner, PITHA 99/4, (1999).

[8] K. Hanke, DESY-TESLA 97-14, (1997). 